\title{
Nova espécie de Theridiosoma (Araneae, Theridiosomatidae) do sul do Brasil
}

\author{
Everton N. L. Rodrigues ${ }^{1} \&$ Ricardo $\mathrm{Ott}^{2}$
}

1. Programa de Pós-Graduação em Biologia Animal, Departamento de Zoologia, Instituto de Biociências, Universidade Federal do Rio Grande do Sul, Av. Bento Gonçalves, 9500, Bloco IV, Prédio 43435, 91501-970 Porto Alegre, RS, Brasil. (enlrodrigues@yahoo.com.br) 2. Museu de Ciências Naturais, Fundação Zoobotânica do Rio Grande do Sul, Rua Dr. Salvador França, 1427, 90690-000 Porto Alegre, RS, Brasil. (rott@fzb.rs.gov.br)

\begin{abstract}
New species of Theridiosoma (Araneae, Theridiosomatidae) from Southern Brazil. Theridiosoma chiripa sp. nov.
\end{abstract} from the states of Rio Grande do Sul and Santa Catarina, Brazil, is described and illustrated.

KEYWORDS. Theridiosomatinae, Theridiosomatidae, Theridiosoma, Neotropical, taxonomy.

RESUMO. Theridiosoma chiripa sp. nov. dos estados do Rio Grande do Sul e Santa Catarina, Brasil, é descrita e ilustrada.

PALAVRAS-CHAVE. Theridiosomatinae, Theridiosomatidae, Theridiosoma, Neotropical, taxonomia.

A família Theridiosomatidae inclui aranhas pequenas, geralmente com menos de $3 \mathrm{~mm}$ de comprimento, que constroem teias orbiculares modificadas (CODDINGTON \& VALERIO, 1980) e habitam principalmente locais escuros e úmidos como cavernas, vegetação junto ao solo de florestas ou áreas alagadas. Sua distribuição é principalmente cosmotropical, com alguns gêneros exclusivamente neotropicais e outros ocorrendo no Velho ou Novo Mundo (CoDdington, 1986), no entanto somente os gêneros Ogulnius O. P.Cambridge, 1882, Theridiosoma O. P.-Cambridge, 1879 e Wendilgarda Keyserling, 1886 ocorrem em ambos.

A família Theridiosomatidae foi estabelecida por VELLARD (1924) que a separou da antiga família Argiopidae. A mais recente revisão da família foi realizada por CODDINGTON (1986), que considera a existência de quatro subfamílias: Platoninae Coddington, 1986; Epeirotypinae Archer, 1953; Ogulniinae Coddington, 1986 e Theridiosomatinae Simon, 1881. Atualmente a família é representada por 12 gêneros e 72 espécies (Platnick, 2004).

A subfamília Theridiosomatinae tem ampla distribuição mundial e conta, atualmente, com cinco gêneros: Andasta Simon, 1895, com quatro espécies; Baalzebub Coddington, 1986, com três; Epilineutes Coddington, 1986, monotípico; Theridiosoma O. P. Cambridge, 1879, com vinte e Wendilgarda Keyserling, 1886, com dez espécies.

O gênero Theridiosoma inclui oito espécies neotropicais; destas, apenas duas ocorrem no Brasil, $T$. concolor Keyserling, 1884, descrita para o Amazonas e conhecida somente a fêmea, porém o holótipo está perdido e T. obscurum (Keyserling, 1886), também somente a fêmea descrita para a localidade de Rio Grande. A espécie-tipo do gênero, T. gemmosum (L. Koch, 1877), foi descrita originalmente para Nuremberg, Alemanha e hoje está distribuída na América do Norte, Europa e Ásia central (Platnick, 2004). O conhecimento sobre o gênero Theridiosoma no Brasil é muito precário, necessitando diversos estudos deste grupo no país.
Os espécimes examinados estão depositados na coleção aracnológica do Museu de Ciências Naturais, Fundação Zoobotânica do Rio Grande do Sul (MCN; E. H. Buckup) e Museu de Ciências e Tecnologia da Pontifícia Universidade Católica do Rio Grande do Sul (MCTP; A. A. Lise), ambos em Porto Alegre. Descrições e a terminologia do palpo seguem CoDDINGTon (1986; 1990). A genitália da fêmea foi clarificada com ácido lático por aproximadamente 30 minutos, até a visualização das estruturas internas. Medidas são apresentadas em milímetros.

\section{Theridiosoma chiripa sp. nov. (Figs. 1-12)}

Material-tipo. Holótipo ${ }^{\prime}$, BRASIL, Rio Grande do Sul: Capão do Leão (Horto Botânico Irmão Teodoro Luís), 07.II.2003, E. N. L. Rodrigues col., pitfall trap (MCN 37597). Parátipos 20', 3ㅇ, mesma localidade do holótipo, 06.I.2003, 24.I.2003, 21.II.2003, E. N. L. Rodrigues col., pitfall trap (MCN 37598, 37599, 37600, 37618); O’, 49 , São Francisco de Paula, Centro de Estudos e Conservação da Natureza Pró-Mata, 14.IV.2002, 09.VIII.2001, R. Ott col., pitfall trap (MCN 37601, 37602); o', 2 , , mesma localidade do holótipo, 24.I.2003, E. N. L. Rodrigues col., pitfall trap (MCTP 16565).

Etimologia. O nome específico em aposição referese a uma parte da indumentária dos índios, posteriormente utilizada pelos gaúchos no Rio Grande do Sul.

Diagnose. O palpo do macho de Theridiosoma chiripa (fig. 4), similar ao de T. gemmosum (L. Koch, 1877) (CoDDington, 1986, figs. 134-137, 138-149; Dondale et al., 2003, figs. 773-778) e T. goodnightorum Archer, 1953 (CoDDington, 1986, figs. 130, 131), distingue-se pela forma do condutor, pela região posterior da apófise média terminando de forma abrupta e pela apófise embólica projetada. A fêmea caracteriza-se pela forma dos ductos copulatórios, distinguindo-se de T. concolor (KEYSERLING, 1884, pr. 10, fig. 133) e T. obscurum (KeYSERLING, 1886, pr. 15, fig. 211) pela forma cônica da borda posterior do epígino (figs. 6-9). 


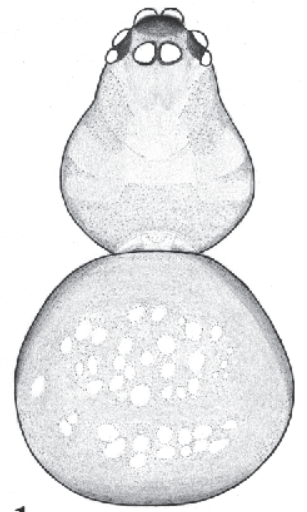

1

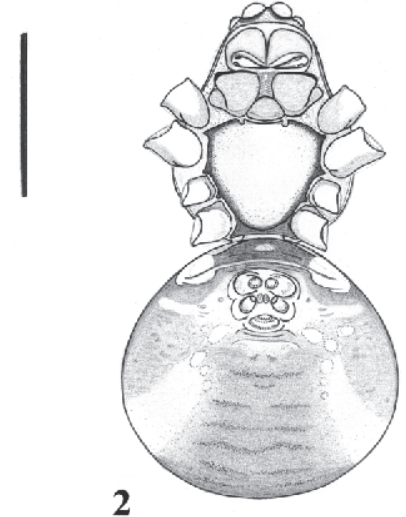

2

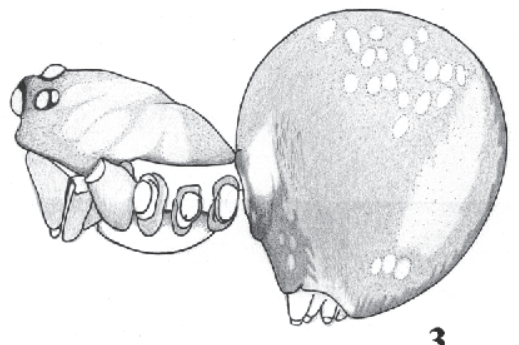

3

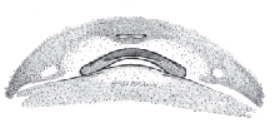

7
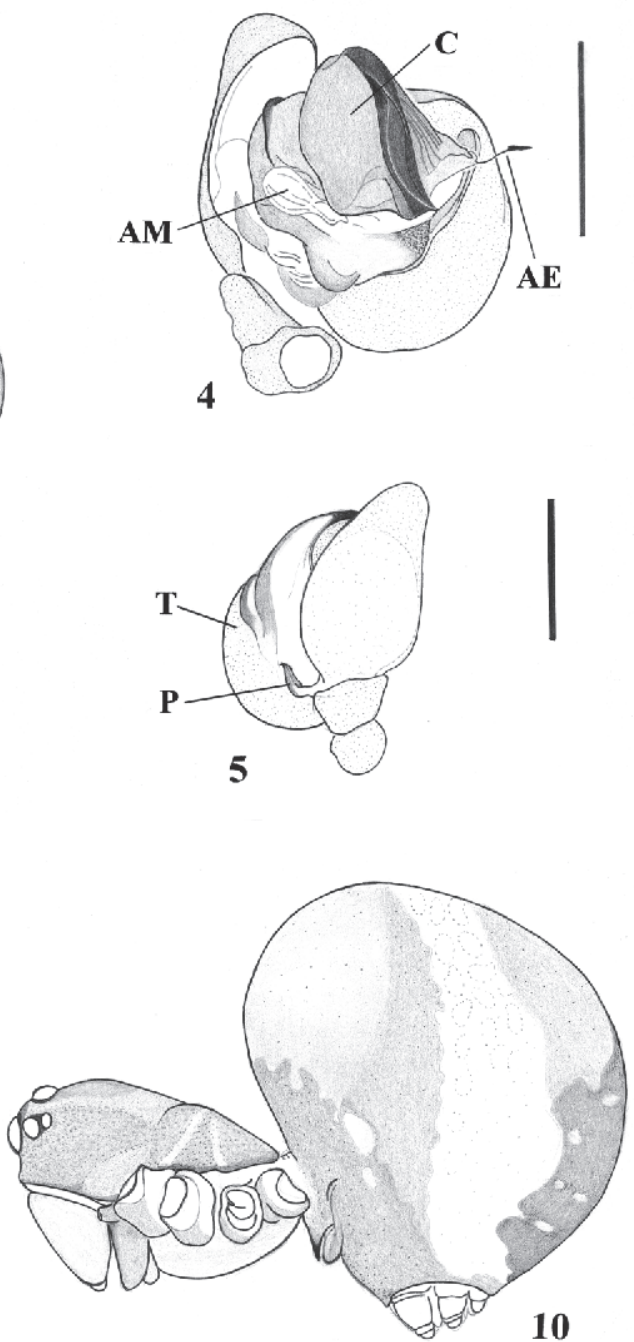

10
8

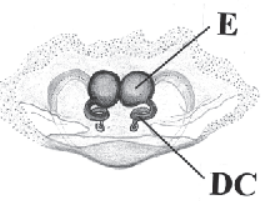

9

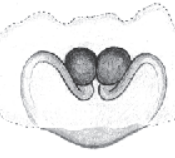

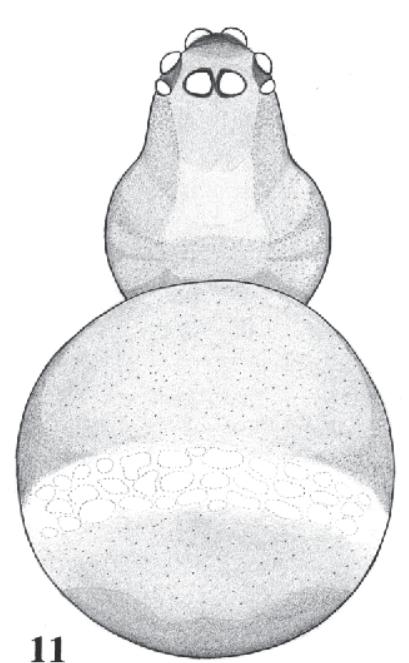

11

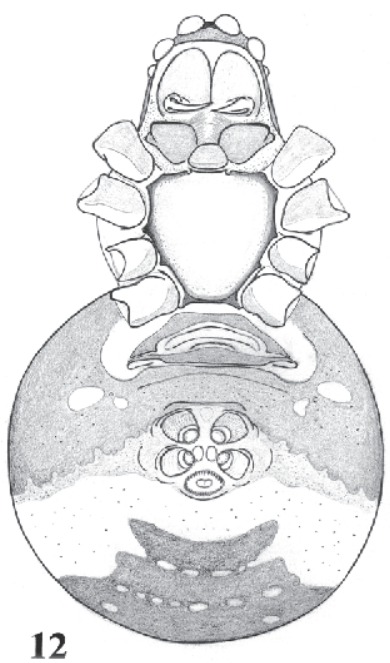

12

Figs. 1-12. Theridiosoma chiripa sp. nov.: 1-3, macho: 1, dorsal; 2, ventral; 3, lateral. 4-5, palpo do macho: 4, ventral; 5, dorsal. 6-9, epígino: 6, ventral; 7, posterior; 8, ventral, clarificado; 9, dorsal, clarificado. 10-12, fêmea: 10, lateral; 11, dorsal; 12, ventral. Abreviaturas: AE, apófise embólica; AM, apófise média; C, condutor; DC, ducto copulatório; E, espermateca; P, paracímbio; T, tégulo. Barras: figs. 1-3, 10-12, 0,5 mm; figs. 4, 5, 0,2 mm; figs. 6-9, 0,25 mm. 
Descrição. Holótipo ơ. Comprimento total 1,57. Carapaça, comprimento 0,77, largura 0,60, região cefálica 0,37 . Esterno, comprimento 0,33, largura 0,35. Clípeo, altura 0,07. Abdômen, comprimento 0,80, largura 0,87, altura 1,07. Pernas 1,2,4,3. Comprimento I/II/III/IV: fêmures $0,71 / 0,57 / 0,31 / 0,43$; patelas $0,20 / 0,22 / 0,15 / 0,21$; tíbias $0,47 / 0,48 / 0,18 / 0,31$; metatarsos $0,43 / 0,37 / 0,25 / 0,25$; $\operatorname{tarsos} 0,25 / 0,25 / 0,18 / 0,18$; total 2,06/1,89/1,07/1,38. Carapaça amarelo-clara acinzentada, mais pigmentada e escura nas laterais e, principalmente, na região ocular (figs. 1, 3). Quelíceras e esterno amarelo-claros, mais escuros nas bordas. Coxas amarelo-acinzentadas, pernas amarelo-claras. Abdômen dorsalmente amarelo-claro, exceto laterais anteriores e região posterior mais escura. Medianamente com pequenos pontos, arredondados e com pigmentação branca. Ventre castanho-escuro na região anterior, com manchas mais escuras na posterior, laterais castanho-claras e amarelo-claras. Área ao redor das fiandeiras amarelo-clara (fig. 2). Olhos heterogêneos, OMA escuros, demais perolados; OMA pouco maiores que os demais e os OLP os menores. Fila ocular posterior pouco mais larga que a anterior. Palpo possui paracímbio rudimentar e tégulo muito desenvolvido (fig. 5).

Parátipo (MCN 37599). Comprimento total, 1,79. Carapaça, comprimento 0,82 , largura 0,52 , região cefálica 0,42 . Esterno, comprimento 0,37, largura 0,35. Clípeo, altura 0,08. Abdômen, comprimento 1,05, largura 1,07, altura 1,30. Pernas 1,2,4,3. Comprimento I/II/III/IV: fêmures 0,61/0,53/ $0,31 / 0,47$; patelas $0,26 / 0,25 / 0,22 / 0,23$; tíbias $0,38 / 0,33 / 0,16 /$ 0,31 ; metatarsos $0,35 / 0,32 / 0,23 / 0,28 ; \operatorname{tarsos} 0,25 / 0,23 / 0,18 /$ 0,21 ; total 1,85/1,66/1,10/1,50. Coloração semelhante a do macho, exceto no dorso do abdômen, com pequenos pontos arredondados esbranquiçados formando uma faixa mediana transversal que se estende ventralmente até as fiandeiras (figs. 10, 11). Ventre castanho-escuro, principalmente na região posterior (fig. 12). Olhos com o mesmo padrão dos machos, exceto que a distância entre a fila ocular anterior e posterior é um pouco menor. Epígino com ductos copulatórios complexos, espermatecas circulares e a borda posterior mais esclerotinizada que o restante da placa epiginal (figs. 8, 9).

Variação. Comprimento total (6 machos): 1,15 a 1,62; (11 fêmeas): 1,47 a 2,12. Os pontos esbranquiçados em algumas fêmeas se distribuem por toda a região dorsal do abdômen, não formando uma faixa transversal. Em ambos os sexos alguns indivíduos apresentam padrão geral de coloração mais claro.

Material examinado. BRASIL, Santa Catarina: Blumenau (2700'31''S; 49 00'66"'W), o, 04.II.1996, A. B. Bonaldo col. (MCN 27283); Ilhota (26 48'29''S; 48 57'19'”), +, 03.II.1996, A. B. Bonaldo col. (MCN 27295). Rio Grande do Sul: São
Francisco de Paula (Centro de Estudos e Conservação da Natureza Pró-Mata), 139, 03.III.2001, 07.IV.2001, 15.XII.2001, 02.II.2002, R. Ott col., pitfall trap (MCN 37603-37606); Cambará do Sul, ㅇ, 26.X.1993, L. A. Moura col. (MCN 24444); Triunfo (Parque Copesul de Proteção Ambiental), , 28.V.2001, M. A. L. Marques col. (MCN 33940); 20', 49, 23.VII.2002, 17.IX.2002, 22.X.2002, 19.XI.2002, R. Ott col. (MCN 34541, 34549, 34725, 34873, 34971); Charqueadas (Reserva Ecológica Ademir Scarpat), $q$, 21.V.2003, R. Ott col. (MCN 35705); Capão do Leão (Horto Botânico Irmão Teodoro Luís), 2+, 21.II.2003, 24.I.2003, E. N. L. Rodrigues col., pitfall trap (MCN 37607, 37619).

História natural. Os espécimes examinados foram coletados em ambientes bastante distintos: mata de restinga e Floresta Ombrófila Mista (mata com araucária); nesta última, a espécie foi registrada em áreas primárias, secundárias e de silvicultura de Pinus spp. (Pinaceae). Nestas áreas, T. chiripa sp. nov. foi observada construindo teias verticais com cerca de 15 $\mathrm{cm}$ de diâmetro, preferencialmente nas samambaias que crescem junto à vegetação arbustiva da floresta, em alturas que variam entre 20 e $40 \mathrm{~cm}$ do solo. Na mata de restinga não foram observadas teias e os indivíduos foram coletados com guarda-chuva entomológico.

Distribuição. Sul do Brasil (Rio Grande do Sul e Santa Catarina).

Agradecimentos. À curadora do MCN, Erica H. Buckup pelo acesso ao material estudado; à Maria Aparecida de L. Marques e aos revisores anônimos pelas sugestões apresentadas.

\section{REFERÊNCIAS BIBLIOGRÁFICAS}

Coddington, J. A. 1986. The genera of the spider family Theridiosomatidae. Smithsonian Contributions to Zoology, 422:1-96.

1990. Ontogeny and homology in the male palpus of orbweaving spiders and their relatives, with comments on phylogeny (Araneoclada: Araneoidea, Deinopoidea). Smithsonian Contributions to Zoology, 496:1-52.

Coddington, J. A. \& Valerio, C. E. 1980. Observations on the web and behavior of Wendilgarda spiders (Araneae: Theridiosomatidae). Psyche, 87:93-105.

Dondale, C. D.; Redner, J. H.; Paquin, P. \& Levi, H. W. 2003. The insects and arachnids of Canada. Part 23. The orb-weaving spiders of Canada and Alaska (Araneae: Uloboridae, Tetragnathidae, Araneidae, Theridiosomatidae). Ottawa, NRC Research Press. 371p.

Keyserling, E. 1884. Die Spinnen Amerikas, Theridiidae. Nürnberg, Bauer \& Raspe. Bd. 2, Hälfte 1. 222p.

. 1886. Die Spinnen Amerikas, Theridiidae. Nürnberg, Bauer \& Raspe. Bd. 2, Hälfte 2. 295p.

Platnick, N. I. 2004. The world spider catalog, version 5.0. American Museum of Natural History. Disponível em: <http:/ /research.amnh.org/entomology/spiders/catalog/index.html>. Acesso em: 08.2004.

Vellard, J. 1924. Études de Zoologie. Archivos do Instituto Vital Brazil, 2:121-170. 